\title{
Nanoscale quantum dot infrared sensors with photonic crystal cavity
}

\author{
K. T. Posani, V. Tripathi, S. Annamalai, N. R. Weisse-Bernstein, and S. Krishna ${ }^{\text {a) }}$ \\ Center for High Technology Materials, ECE Department, University of New Mexico, 1313 Goddard St., SE, \\ Albuquerque, New Mexico 87106 \\ R. Perahia, O. Crisafulli, and O. J. Painter \\ Department of Applied Physics, 1200 East California Boulevard, California Institute of Technology, \\ Pasadena, California 91125
}

(Received 14 November 2005; accepted 18 March 2006; published online 12 April 2006)

\begin{abstract}
We report high performance infrared sensors that are based on intersubband transitions in nanoscale self-assembled quantum dots combined with a microcavity resonator made with a high-index-contrast two-dimensional photonic crystal. The addition of the photonic crystal cavity increases the photocurrent, conversion efficiency, and the signal to noise ratio (represented by the specific detectivity $D^{*}$ ) by more than an order of magnitude. The conversion efficiency of the detector at $V_{b}=-2.6 \mathrm{~V}$ increased from $7.5 \%$ for the control sample to $95 \%$ in the PhC detector. In principle, these photonic crystal resonators are technology agnostic and can be directly integrated into the manufacturing of present day infrared sensors using existing lithographic tools in the fabrication facility. (C) 2006 American Institute of Physics. [DOI: 10.1063/1.2194167]
\end{abstract}

Infrared sensors in the wavelength range of $3-25 \mu \mathrm{m}$ are of immense technological importance due to their application in medical diagnostics, fire-fighting equipment, and night vision systems. Quantum dot infrared photodetectors have been identified as an emerging technology for this wavelength regime due to their low dark current leading to a potentially higher operating temperature and normal incidence operation based on a mature GaAs technology. ${ }^{1-5}$ Presently, high performance midinfrared detectors are based on mercury cadmium telluride (MCT). Due to a dramatic change of the band gap as a function of material composition, it is very challenging to reproducibly obtain large area homogeneous materials suitable for large area focal plane arrays (FPA) based on this material system. In contrast, mature materials growth technologies for III-V semiconductors can provide very accurate control of compositions and homogeneity. Therefore there is interest in developing IR photodetectors using III-V materials. One of the most promising III-V semiconductor long wavelenght infrared (LWIR) detectors is the quantum well infrared photodetector (QWIP), ${ }^{6-9}$ which employs the intersubband or the subbandto-continuum transitions in quantum wells. One of the drawbacks of $n$-type QWIPs is that they cannot detect normally incident light due to the restriction of selection rules for the optical transition. In contrast, the intersubband optical transitions in quantum dots (QDs) do not have that restriction, due to the three-dimensional quantum confinement. Theoretically, quantum dot infrared photodetectors (QDIPs) and quantum dot-in well (DWELL) detectors (which is a combination of a quantum dot and quantum well detector) offer several advantages over QWIPs, including lower dark current (hence higher $T$ operation), higher responsivity, normal incidence detection, and improved radiation hardness. ${ }^{10,11}$ QDIPs with low dark current densities and high operating temperature have been reported. ${ }^{2,3}$ Asymmetrically designed DWELL detectors have also been shown to have a biasdependent spectral response that is suitable for multispectral

\footnotetext{
${ }^{a}$ Author to whom correspondence should be addressed; electronic mail:
} skrishna@chtm.unm.edu imagery. ${ }^{12}$ Recently, a two color $320 \times 256$ FPA, based on a voltage-tunable InAs/InGaAs/GaAs DWELL structure has also been demonstrated. ${ }^{13}$ However, the external quantum efficiency (QE), defined as the number of photoelectrons generated per incident photon, and the conversion efficiency (product of the photoconductive gain and $\mathrm{QE}$ ) of the QD detectors have been pretty low $(1 \%-5 \%)$. One of the main reasons for this is that the growth of self-assembled QDs needs sufficient strain in the QD regions, making it challenging to grow a thick active layer without causing misfit dislocations, and hence not providing sufficient absorption of the signal light. In this letter, we report the fabrication of a photonic crystal QD detector (PhC-QD) in which a twodimensional hexagonal $\mathrm{PhC}$ is used as an optical resonator to improve the conversion efficiency of the sensor. Thus without using a vertical cavity (as done in a resonant cavity enhanced detector with distributed Bragg reflectors), the coupling efficiency of the light with the active region is increased. Simply viewed, the $\mathrm{PhC}$ represents a regular array of holes that is used to modify the local refractive index to provide localized modes in the "photonic" band structure. ${ }^{14-16}$ The PhC has a grating effect that "diffracts" the normally incident radiation to the in-plane direction. The in-plane radiation then propagates extremely slowly at the $\Gamma$ point of the band structure, resulting in an increased interaction of the incident light with the active region (represented by the quantum dots). A schematic and an image of a hexagonal $\mathrm{PhC}$ are shown in Fig. 1. Using this approach, we have obtained the highest reported conversion efficiency in QD detectors $\left(\sim 95 \%\right.$ at $\left.V_{b}=-2.6 \mathrm{~V}\right)$. It should be noted that this approach is detector agnostic and can be applied to any detector in which the fabrication of holes does not lead to an increase in the surface recombination current. Another advantage of this approach is that it can be easily incorporated into the FPA fabrication process of present day infrared sensors, since the holes $(2-3 \mu \mathrm{m}$ in diameter for $\lambda$ $\sim 8-10 \mu \mathrm{m})$ can be defined using conventional optical lithography. Moreover, with a straightforward modification, a single or multielement defect can be introduced in the $\mathrm{PhC}$, thereby selectively increasing the response of photons with a specific energy. ${ }^{17}$ Thereby by changing the dimensions of the 


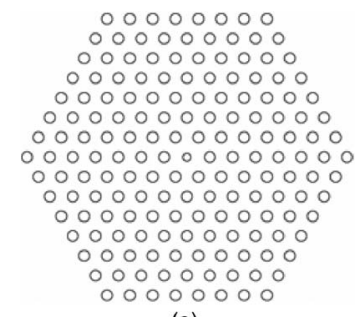

(a)

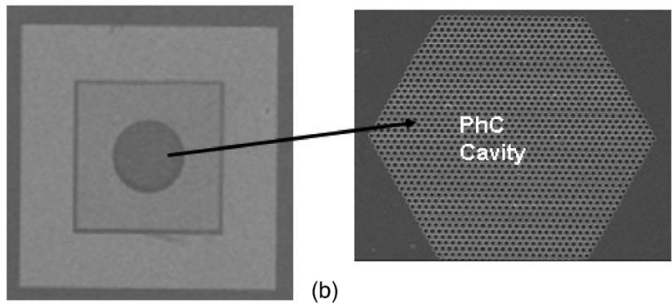

FIG. 1. (a) Photonic crystal resonant cavity comprising of hexagonal pattern of air holes. (b) Image of a PhC defined in a quantum dot detector.

defect, the resonance wavelength can be altered leading to the fabrication of a spectral element in each pixel of the FPA. This would have a revolutionary impact on multispectral imaging (MSI) and hyperspectral imaging (HSI) detectors.

The detectors were grown by solid source molecular beam epitaxy (MBE), and the details are listed elsewhere. ${ }^{1,13}$ The detector structure consists of a 15 stack asymmetric DWELL structure sandwiched between two highly doped $n$-GaAs contact layers, grown on a semi-insulating GaAs

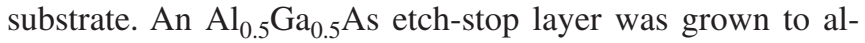
low substrate removal in the FPA process. The DWELL region consists of a 2.4 monolayer deposition of $n$-doped InAs QDs in an $\mathrm{In}_{0.15} \mathrm{GaAs}_{0.85}$ As well, itself placed in GaAs. The well widths below and above the dots are 50 and $60 \AA$, respectively. The well width asymmetry, combined with the inherent asymmetry associated with QD formation, leads to a voltage-tunable spectral response in the finished devices. ${ }^{13}$ Standard processing techniques were used to fabricate topilluminated $400 \times 400 \mu \mathrm{m}^{2}$ test pixels, with aperture diameters ranging between 25 and $300 \mu \mathrm{m}$.

Hexagonal $\mathrm{PhC}$ cavities were designed, and their band structure was modeled. The first step in the $\mathrm{PhC}$ modeling is to find the effective index of the TE and TM fundamental modes of the unpatterned quantum dot heterostructure using finite-difference techniques. ${ }^{18}$ Plane wave expansion methods are then used with the calculated effective index to analyze the band structure of the waveguide. The cavity modes

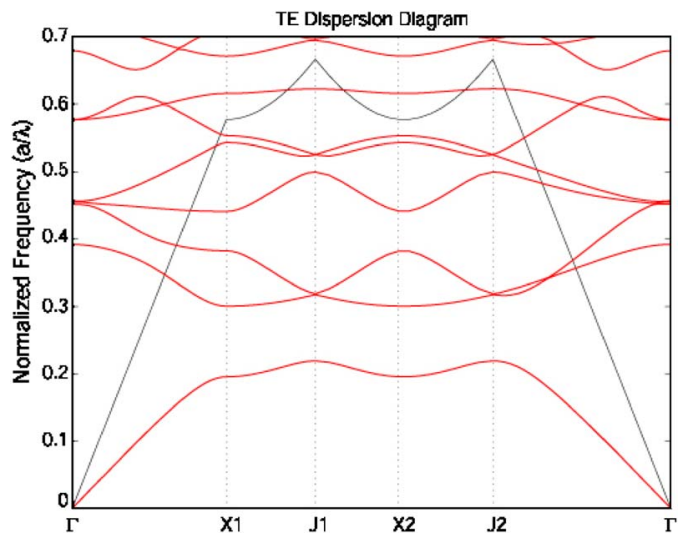

FIG. 2. The TE dispersion diagram showing the normalized frequency at high symmetry points in the Brillouin zone. Downloaded 06 May 2006 to 131.215.240.9. Redistribution subject to AIP license or copyright, see http://apl.aip.org/apl/copyright.jsp
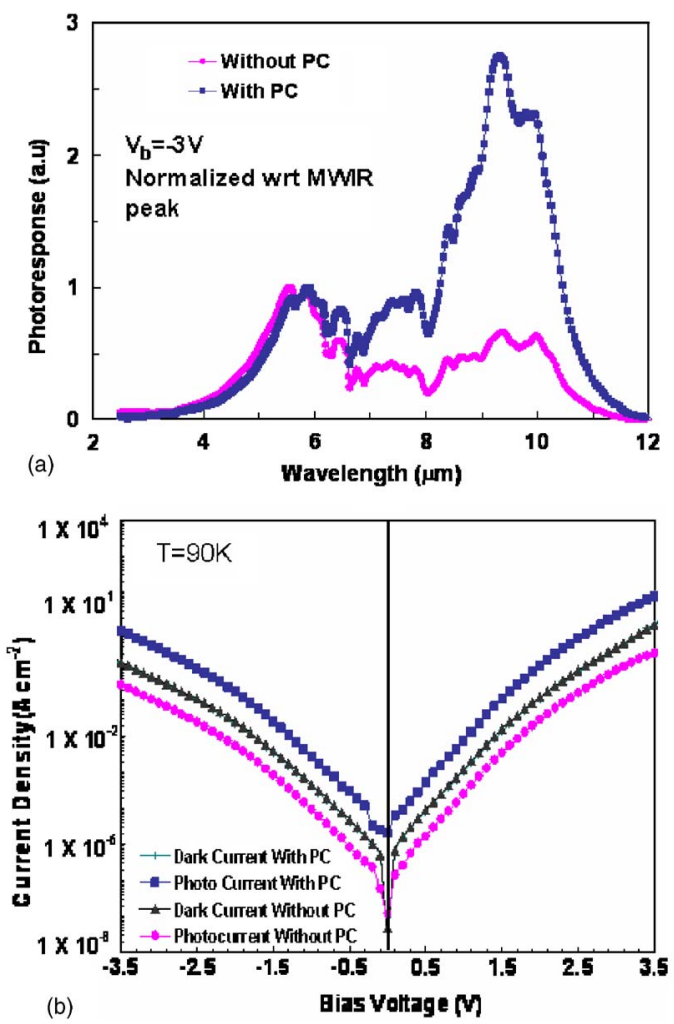

FIG. 3. (a) Comparison of the spectral response plots of the DWELL detectors with and without the photonic crystal cavity at a bias of $-3 \mathrm{~V}$. (b) Photo current and dark current densities of the normal and photonic crystal detectors at $90 \mathrm{~K}$. The photocurrent density was measured with the detector viewing a $300 \mathrm{~K}$ scene in a F1.7 configuration.

are tuned by means of two geometric parameters of the photonic crystal pattern, namely, the lattice spacing $a$ and the hole radius $r$. From the band structure analysis certain high symmetry points in the Brillouin zone are targeted due to the flat band (large density of optical states) nature of the photonic bands. In particular, the optical modes near the $\Gamma$ point of the Brillouin zone are targeted due to their efficient coupling to normal incident light. The high-index contrast of the photonic crystal structure employed in this work provides a significantly wide range of optical frequencies and in-plane wave vectors (equivalently a large range of incident angles from the air) around the $\Gamma$ point for which there exists a high density of optical modes (flatband characteristics). For achieving hyperspectral response, localized defect modes can also be introduced into the photonic lattice by perturbing the radius of the air holes at some desired locations in the hexagonal lattice. The localized defect mode normalized frequency for the structures fabricated in this work occurs approximately at $a / \lambda \sim 0.3$ from the finite difference time domain simulations. For a desired wavelength of $\lambda$ $=8.1 \mu \mathrm{m}$ this results in a lattice spacing of $2.4 \mu \mathrm{m}$. Figure 2 shows the band structure and localized modes in a PhC cavity. $\mathrm{PhCs}$ were then defined using e-beam lithography (although it should be mentioned that these patterns can be terns were etched into the active region, and a finished device is shown in Fig. 1.

Bias-dependent spectral response curves for a pixel with a $300 \mu \mathrm{m}$ diameter aperture with and without a PhC, measured using a Nicolet 870 Fourier transform infrared spectrometer (FTIR), at a detector bias of $-3 \mathrm{~V}$ at $50 \mathrm{~K}$, are shown in Fig. 3(a). The spectra contain peaks centered in generated using conventional optical lithography). The pat- 

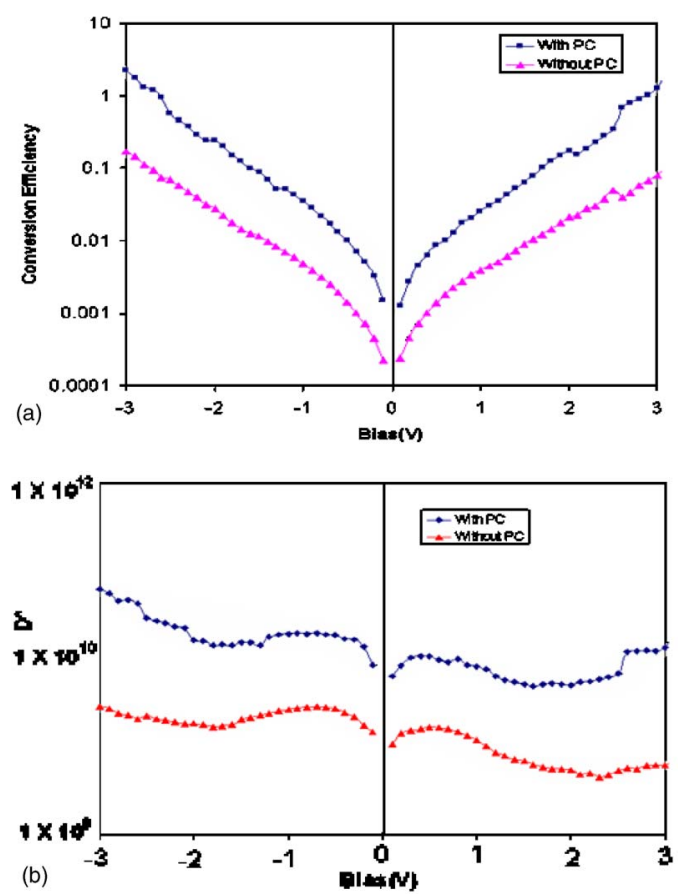

FIG. 4. Comparison of the conversion efficiency and detectivity $\left(D^{*}\right)$ of the detectors with and without $\mathrm{PhC}$.

around 6 and $10 \mu \mathrm{m}$. The shorter wavelength $(\sim 6 \mu \mathrm{m})$ peak is dominant at smaller bias voltages $\left(\left|V_{b}\right|<2 \mathrm{~V}\right)$, whereas the longer wavelength $(\sim 10 \mu \mathrm{m})$ peak is dominant at higher biases $\left(\left|V_{b}\right|>2 \mathrm{~V}\right)$. We believe that the $10 \mu \mathrm{m}$ peak arises from transitions from the ground state in the dot to a low lying states in the quantum well $(\mathrm{QW})$, whereas the $6 \mu \mathrm{m}$ peak arises due to transitions from the ground state of the dot to a higher lying state in the well. This also explains the appearance of the LWIR peak at increased bias since the carriers in the lower lying state are extracted by field assisted tunneling, a process that dominates at higher biases. The peak positions are independent of temperature, although the ratio of the peak heights varies as a function of temperature. It is interesting to note that the spectral response of the device with the $\mathrm{PhC}$ is a factor of 5 higher than that of the control sample, indicating strong coupling of the photonic crystal with the QDs. However, we do not see any pronounced amplification of certain peaks in the spectrum indicating that the defect mode is not strongly coupled.

The dark current density characteristics of the devices at $90 \mathrm{~K}$ are shown in Fig. 3(b). The $300 \mathrm{~K}$ background photocurrent density under F1.7 (field of view $=33^{\circ}$ ) is also shown. Note that there is an order of magnitude increase in the photocurrent density in the device with $\mathrm{PhC}$ with no change in the dark current density. This indicates that there is no significant effect of surface states on the performance of the device. This could be due to the fact that the carriers are localized in the dots and do not feel the effect of the surfaces. It is to be noted that the fraction of material removed by the $\mathrm{PhC}$ processing is about $47 \%$ of the aperture area and was not included in the calculation. Thus, this measurement underestimates the increase in the photocurrent density. Noise spectra and responsivity measurements were undertaken using a calibrated blackbody at $800 \mathrm{~K}$. The signal (photocurrent) was amplified by a SRS 570 low noise current amplifier and then displayed by a SRS 760 fast Fourier transform (FFT) spectrum analyzer. The conversion efficiency and de- tectivity obtained from the test devices at $78 \mathrm{~K}$ are shown in Fig. 4. It is to be noted that the conversion efficiency and the detectivity (measure of signal to noise ratio) increase by more than an order of magnitude in the presence of the PhC. The conversion efficiency at $V_{b}=-2.6 \mathrm{~V}$ (for a peak wavelength around $9 \mu \mathrm{m}$ ) is $95 \%$ in the $\mathrm{PhC}$ detector as opposed to $7.5 \%$ for the baseline detector. These are the highest conversion efficiencies reported in quantum dot detectors. Although we have not measured a gain in these devices, gain has been reported in QD devices. ${ }^{19}$ Presently we are trying to incorporate these $\mathrm{PhC}$ cavities in focal plane arrays.

In conclusion we report the design and fabrication of a photonic crystal $(\mathrm{PhC})$ quantum dot $(\mathrm{PhC}-\mathrm{QD})$ sensor. By incorporating a $\mathrm{PhC}$ resonant cavity, the interaction length between the photons and electrons was increased leading to an order of magnitude increase in the photocurrent, conversion efficiency, and detectivity of the sensor. These PhC cavities are detector agnostic and can be incorporated in to the fabrication scheme of present day infrared sensors. This represents a major breakthrough in sensor research since this will lead to a dramatic improvement in the performance of infrared imaging systems, which are in great need for a variety of civilian and military applications.

The authors would like to acknowledge support from NSF ECS Grant Nos. 0428756, 0401154, and 0434102 and the AFOSR MURI Grant on Plasmonics.

${ }^{1}$ S. Krishna, J. Phys. D 38, 2142 (2005).

${ }^{2}$ A. D. Stiff, S. Krishna, P. Bhattacharya, and S. Kennerly, Appl. Phys. Lett. 79, 421 (2001).

${ }^{3}$ E. T. Kim, A. Madhukar, Z. Ye, and J. C. Campbell, Appl. Phys. Lett. 84, 3277 (2004)

${ }^{4}$ J. Phillips, J. Appl. Phys. 91, 4590 (2002).

${ }^{5}$ M. Razeghi, W. Zhang, H. C. Lim, S. Tsao, J. Szafraniec, M. Taguchi, and B. Movaghar, Proc. SPIE 5838, 125 (2005).

${ }^{6}$ S. D. Gunapala, S. V. Bandara, J. K. Liu, E. M. Luong, N. Stetson, C. A. Shott, J. J. Bock, S. B. Rafol, J. M. Mumolo, and M. J. McKelvey, IEEE Trans. Electron Devices 47, 326 (2000).

${ }^{7}$ J. Jiang, K. Mi, S. Tsao, W. Zhang, H. Lim, T. O'Sullivan, T. Sills, M. Razeghi, G. J. Brown, and M. Z. Tidrow, Appl. Phys. Lett. 84, 2232 (2004).

${ }^{8}$ S. D. Gunapala, S. V. Bandara, A. Singh, J. K. Liu, S. B. Rafol, E. M. Luong, J. M. Mumolo, N. Q. Tran, D. Z. Y. Ting, J. D. Vincent, C. A. Shott, J. Long, and P. D. LeVan, IEEE Trans. Electron Devices 47, 963 (2000).

${ }^{9}$ B. F. Levine, J. Appl. Phys. 74, R1 (1993).

${ }^{10}$ V. Ryzhii, Semicond. Sci. Technol. 11, 759 (1996).

${ }^{11}$ P. Bhattacharya, X. H. Su, S. Chakrabarti, G. Ariyawansa, and A. G. U. Perera, Appl. Phys. Lett. 86, 191106 (2005).

${ }^{12}$ U. Sakoğlu, J. S. Tyo, M. M. Hayat, S. Raghavan, and S. Krishna, J. Opt. Soc. Am. B 21, 7 (2004).

${ }^{13}$ S. Krishna, D. Forman, S. Annamalai, P. Dowd, P. Varangis, T. Tumolillo, A. Gray, J. Zilko, K. Sun, M. Liu, J. Campbell, and D. Carothers, Appl. Phys. Lett. 86, 193501 (2005).

${ }^{14}$ O. Painter, R. K. Lee, A. Yariv, A. Scherer, J. D. O. Brien, P. D. Dapkus, and I. Kim, Science 284, 1819 (1999).

${ }^{15}$ K. Inoue and K. Ohtaka, Photonic Crystals Physics, Fabrication, and Applications (Springer, New York, 2004).

${ }^{16}$ S. G. Johnson and J. D. Joannopoulos, Photonic Crystals: The Road from Theory to Practice (Kluwer Academic, Boston, 2002).

${ }^{17}$ R. Colombelli, K. Srinivasan, M. Troccoli, O. Painter, C. F. Gmach, D. M. Tennant, A. M. Sergent, D. L. Sivco, A. Y. Cho, and F. Capasso, Sciencexpress 10, 1090561 (2003).

${ }^{18}$ L. A. Coldren and S. W. Corzine, Laser Diode and Photonic Integrated Circuits (Wiley, New York, 1995).

${ }^{19}$ B. Kochman, A. D. Stiff-Roberts, S. Chakrabarti, J. D. Phillips, S. Krishna, J. Singh, and P. Bhattacharya, IEEE J. Quantum Electron. 39, 459 (2003). 